\title{
Visualization of the Interface between Cell and Ceramic Coating by Electron Microscopy and Tomography Techniques
}

\author{
J. KarbowniczeK*, A. Gruszczyński, A. Kruk and A. Czyrska-Filemonowicz
} International Centre of Electron Microscopy for Materials Science and Faculty of Metals Engineering and Industrial Computer Science, AGH University of Science and Technology, Al. A. Mickiewicza 30, PL-30059 Krakow, Poland

To improve the stability of bone-material connection various surface modifications are applied to titanium based implants. Such new biomaterials require detailed characterization, including their biocompatibility. In the present study focused ion beam-scanning electron microscope tomography was applied to investigate the internal structure of ceramic coating as well as cell-material interface. Focused ion beam-scanning electron microscope tomography involves repeated focused ion beam milling and subsequent scanning electron microscope imaging of exposed sample surface and is followed by 3D reconstruction. This approach enables receiving additional information about the sample, unavailable at $2 \mathrm{D}$ images. The qualitative and quantitative investigation of pores in the ceramic coating was performed. The porosity of investigated volume: $8.5 \times 1.1 \times 3.9 \mu \mathrm{m}^{3}$ was calculated as $24 \%$. Additionally, based on $3 \mathrm{D}$ reconstruction the visualization of the coherent interface between cell and ceramic coating was done.

DOI: 10.12693/APhysPolA.131.1357

PACS/topics: 68.37.Hk; 81.65.Mq; 87.17.Rt; 87.85.J-

\section{Introduction}

Growing demand for novel biomaterials applied in medical field is a driving force for extensive research in this area. Therefore new materials are developed and various manufacturing methods are applied to obtain implants with desired properties. In terms of replacing human tissues by artificial organs the surface characteristics of biomaterials are crucial $[1,2]$. The interactions between leaving cells and material surface can guide organization and growth of tissues around the implant determining its performance [3].

In orthopedic and dental applications titanium alloys are predominant materials for implants staying in direct contact with bone tissue. They have a beneficial combination of mechanical properties, corrosion resistance and biocompatibility. However, for direct, stable and long lasting anchorage of titanium based implant in the bone, surface modification is needed to improve its bioactivity [1]. Among many other methods, micro-arc oxidation (MAO) enables to deposit well adherent, rough and hydroxyapatite (HA) containing coatings on titanium alloys $[4,5]$. MAO is a technique that operates at potentials above the breakdown voltage of an oxide film growing on the metallic sample and is characterized by multiple arcs moving rapidly over the treated surface [6]. Therefore, obtained coatings have characteristic microstructure with crater-like open pores. With careful selection of process parameters and electrolyte composition it is possible to deposit HA in one step process, without subsequent heat treatment $[4,5]$. Mineral part of the bone is mainly composed of carbonated apatite. Thus, application of HA in

*corresponding author; e-mail: jkarbow@agh.edu.pl a form of a coating on metallic implant promotes osseointegration of such prosthesis $[1,5]$.

The comprehensive evaluation of novel or modified biomaterials, also in terms of biocompatibility, is required to verify their applicability. Focused ion beam-scanning electron microscope (FIB-SEM) tomography, commonly used in materials science [7] is being applied in biomaterials research [8] as well as to investigate cell-substrate interactions [2, 3]. In FIB-SEM tomography, by FIB sample is milled layer-by-layer and the exposed surface is imaged by SEM at each stage. Collected 2D images are subsequently used for 3D reconstruction of investigated sample volume $[2,3,7]$. Proper preparation of biological samples is essential while applying electron microscopy techniques to preserve delicate internal cell components and to avoid investigation of artifacts. Various protocols are mentioned in the literature, most of them include: chemical fixation followed by dehydration and sputtering with conductive nanolayer $[9,10]$. Additionally, environmental scanning electron microscope (ESEM) can be applied to image biological specimens in hydrated state, however not for all samples it is suitable [11].

In this work, FIB-SEM tomography was used to investigate the network of internal pores in the coating deposited by MAO on Ti6Al7Nb alloy. Furthermore, on coated samples MG-63 line osteoblast-like cells were in vitro cultured. The interface between cell and ceramic coating was visualized in 3D.

\section{Materials and methods}

\subsection{Micro-arc oxidation process}

Two phase $(\alpha+\beta)$ titanium alloy Ti6Al7Nb (Böhler Edelstahl GmbH, Germany) was used as a substrate in the MAO process. The MAO process parameters were as follows: power supply with alternating current, applied voltage $400 \mathrm{~V}$ (positive half cycle), 
$80 \mathrm{~V}$ (negative half cycle), frequency $250 \mathrm{~Hz}$, the deposition time $5 \mathrm{~min}$, temperature maintained below $30^{\circ} \mathrm{C}$, electrolyte containing: $\mathrm{Na}_{2} \mathrm{HPO}_{4}\left(0.05 \mathrm{~mol} / \mathrm{dm}^{3}\right)$ and $\left(\mathrm{CH}_{3} \mathrm{COO}\right)_{2} \mathrm{Ca} \cdot \mathrm{H}_{2} \mathrm{O}\left(0.1 \mathrm{~mol} / \mathrm{dm}^{3}\right)$ dissolved in distilled water.

Cross-section from the coated $\mathrm{Ti} 6 \mathrm{Al} 7 \mathrm{Nb}$ sample was prepared to facilitate FIB-SEM tomography, which was performed on flat section instead of rough coating surface. Cut sample was placed in cold mounting resin LevoCit (Struers, Poland). After solidification it was grinded, polished, and carbon sputtered.

\subsection{Cell culture}

Prior to cell culture a coated Ti6Al7Nb sample was sterilized in $70 \%$ ethanol and air dried in a sterile laminar flow chamber. The $1 \mathrm{ml}$ of medium containing human osteoblast-like MG-63 cells (Sigma-Aldrich, Poland) in a concentration of 50000 cells per $\mathrm{ml}$ was added to the sample and cultured at $37^{\circ} \mathrm{C}$ for 3 days. Culture media consisting of: eagle's minimum essential media (EMEM) supplemented with 2\% L-glutamine, 1\% non essential amino acids, 10\% fetal bovine serum (FBS) and $2 \%$ penicillin/streptomycin (all reagents Sigma-Aldrich, Poland) was used. During the incubation period the media were replaced every day. After 3 days of culture the cells were fixed with $2.5 \%$ glutaraldehyde, dehydrated in series of ethanol solutions $(30 \%, 50 \%, 70 \%, 96 \%)$, and additionally the sample was submerged in hexamethyldisilazane (Sigma-Aldrich, Poland) to replace remaining water with solvent. Electron microscopy investigations were performed on gold sputtered sample.

\subsection{FIB-SEM tomography}

Tomography investigation was performed with a FIB-SEM NEON CrossBeam 40 EsB of ZEISS (Germany) for two samples: coating deposited by MAO on titanium based alloy — to investigate internal structure of the coating; sample after the in vitro cell culture to investigate cell-coating interactions. The protective platinum layer (Fig. 1) was deposited on the area of interest of each sample surface in order to prevent the occurrence of the curtaining effect, i.e. the irregular removal of material [7]. To provide undisturbed view during sectioning, material surrounding the area chosen for tomography was removed by FIB milling, as shown in Fig. 1. For both samples Ga-ion milling parameters were adjusted to: beam current of $200 \mathrm{pA}$ and voltage of $30 \mathrm{kV}$. Every time after the layer was removed by FIB the exposed surface was imaged by secondary electrons (SE) and backscattered electrons (BSE). The slice thickness for the coating was $22 \mathrm{~nm}$ and that for coating with cell was $20 \mathrm{~nm}$. Collected SEM images were used for $3 \mathrm{D}$ reconstruction, done by ImageJ $1.44 \mathrm{p}$ software.

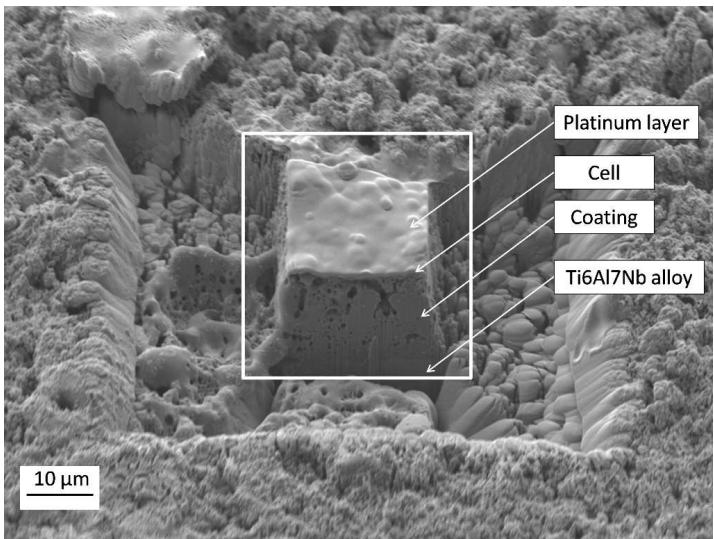

Fig. 1. Area of interest (marked with rectangle) prepared for FIB-SEM tomography.

\section{Results and discussion}

MAO coatings deposited from electrolyte containing $\mathrm{Ca}$ and $\mathrm{P}$ ions were previously studied [12]. Their chemical composition was characterized by X-ray diffraction (XRD) and energy dispersive X-ray spectroscopy (SEMEDS). Three distinct sub-layers were defined, as presented in Fig. 2. Directly at the Ti6Al7Nb substrate the sub-layer of titanium dioxides $\left(\mathrm{TiO}_{2}\right)$ is formed. Subsequently dense, mainly amorphous area containing $\mathrm{TiO}_{2}$ and $\mathrm{CaTiO}_{3}$ is present. Finally, the outer part of the coating is composed of hydroxyapatite (HA) crystals.
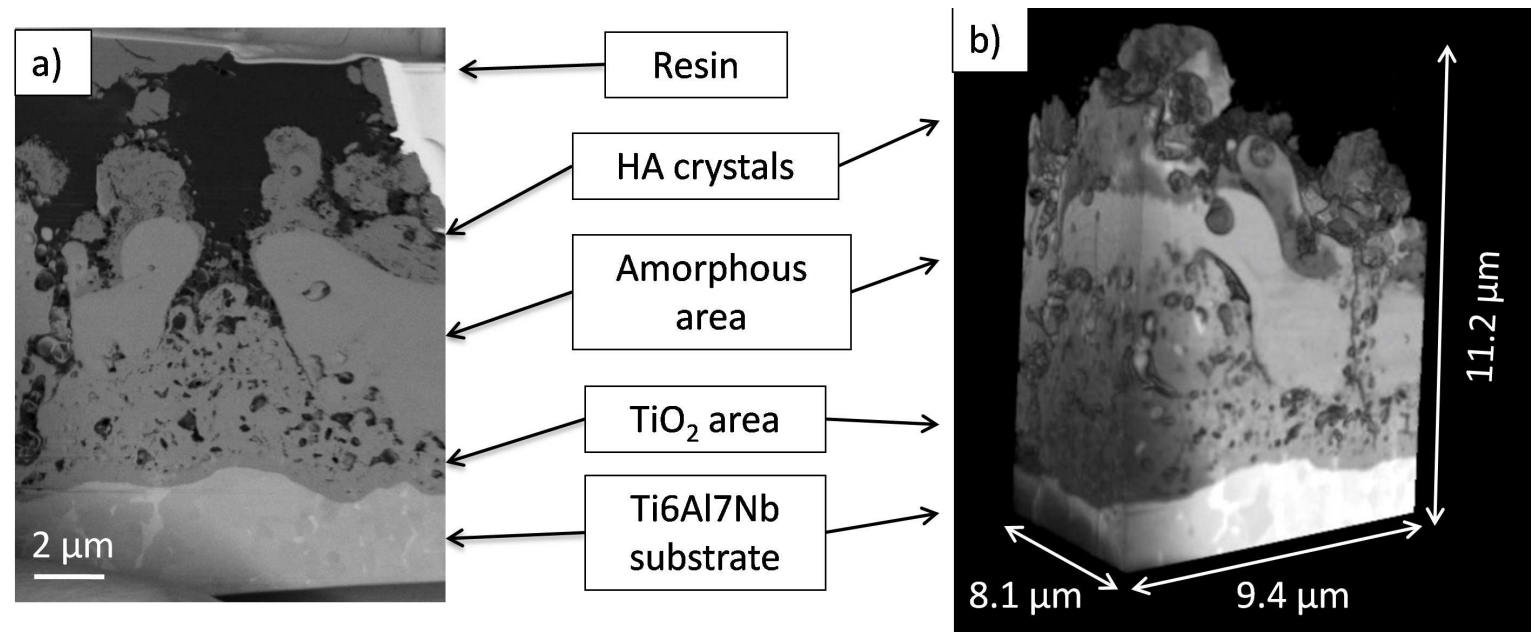

Fig. 2. Location of sub-layers in the coating: (a) example of cross-section image captured during FIB-SEM tomography,

(b) $3 \mathrm{D}$ reconstruction of analysed coating volume after FIB-SEM tomography. 


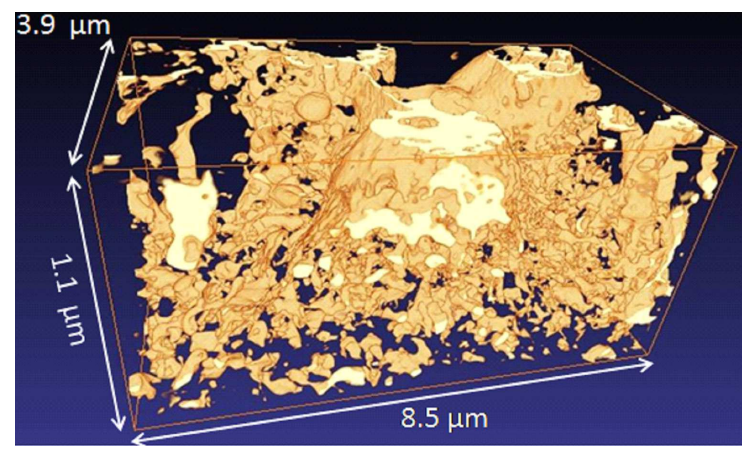

Fig. 3. visualization of the network of internal pores in the coating.

Energy selective backscattered (ESB) detector provides compositional contrast ( $Z$-contrast), therefore it is possible to distinguish different phases present in the sample. At the gray-scale SEM image pores (voids) occurring in the coating are black, whereas different areas of the sample are represented by grey colour varying in the brightness which depends on the composition of particular sub-layer. All sub-layers present in the coating have different chemical composition, therefore, they can be easily distinguished at $2 \mathrm{D}$ images as well as at $3 \mathrm{D}$ tomographic reconstructed volume (Fig. 2). By inverting the images grey-scale it was possible to extract and visualize the network of pores in investigated coating volume (Fig. 3). Porosity of the coating can be estimated based on SEM-BSE image analysis [13]. In case of this study porosity was estimated based on series of SEM-BSE images obtained during FIB-SEM tomography. First step was conversion of the inverted grey-scale images to binary images by thresholding using ImageJ $1.44 \mathrm{p}$ software. It was obtained by turning all pixels below the threshold to black in binary image, and all pixels above the threshold to white in binary image. The threshold was set up to differentiate between pores and all phases present in the sample.
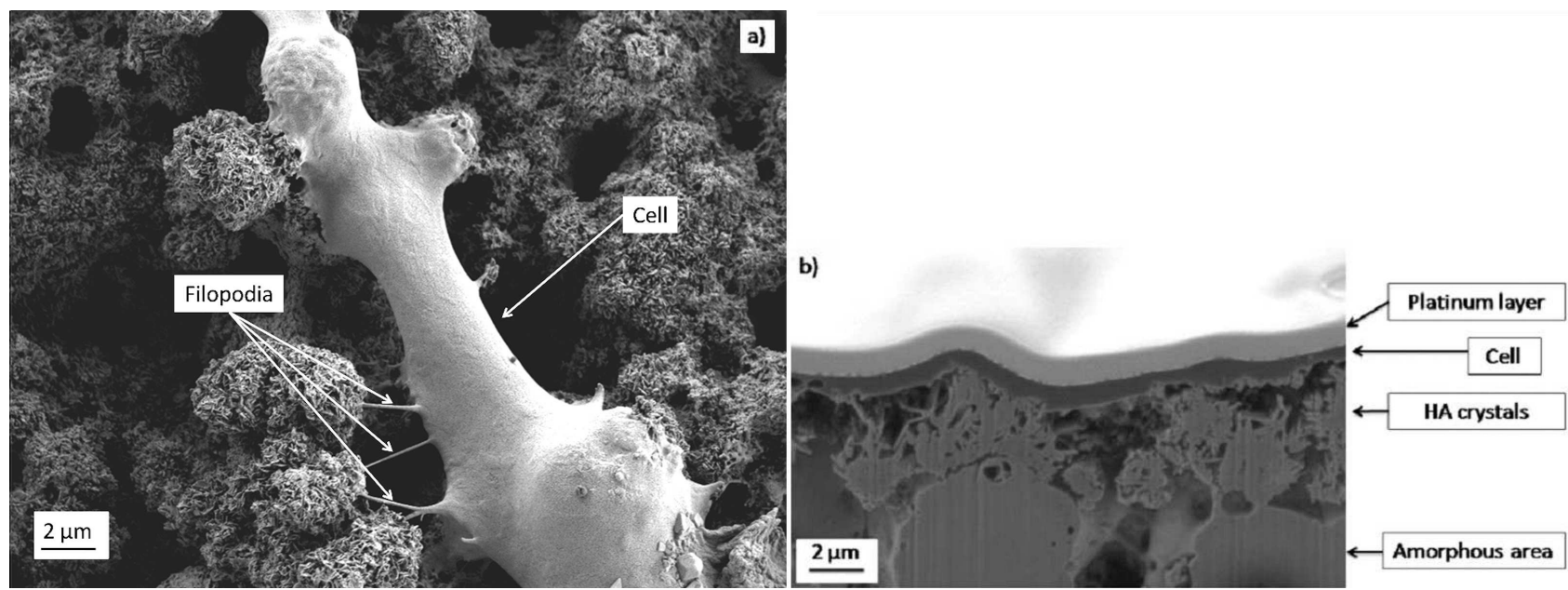

Fig. 4. SEM images of cells on the ceramic coating: (a) top view showing cell attached to the surface, (b) example of cross-section image captured during FIB-SEM tomography.
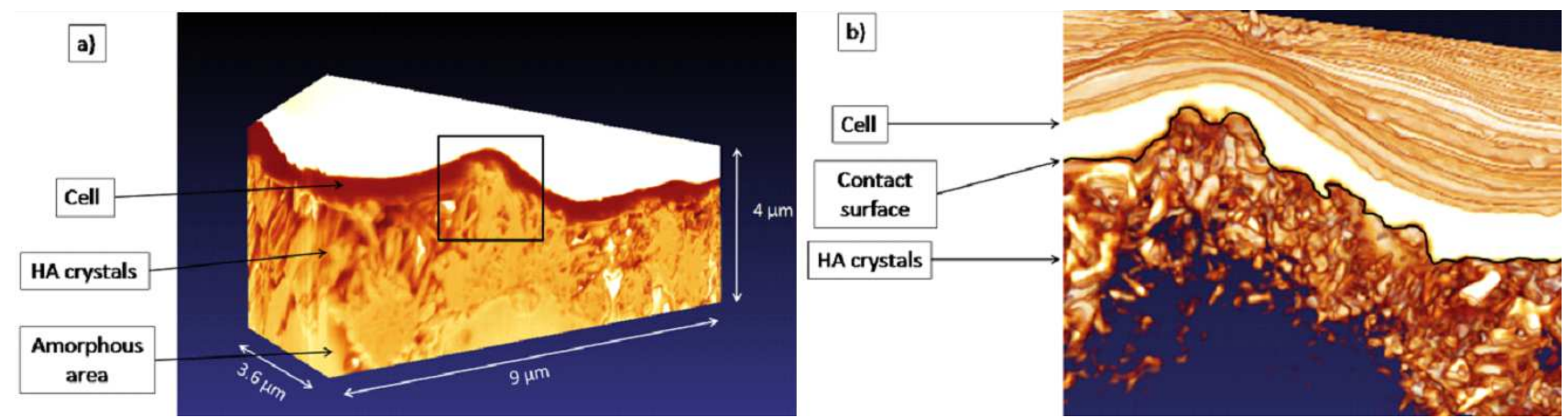

Fig. 5. 3D reconstruction of the cell-material interface: (a) investigated total volume of $9 \times 3.6 \times 4 \mu \mathrm{m}$, (b) selected area (marked with black rectangle in Fig. 5a) showing the coherent interface between cell and HA crystals (marked with black line).

The total relative volume $\left(V_{v}\right)$ of the pores in the analysed volume $(8.8 \times 1.1 \times 3.9 \mu \mathrm{m})$ was $V_{v}=24 \%$. Porosity as well as pores size and distribution might be controlled by MAO process parameters [8]. From the 3D recon- struction numerous, bubble-like pores with a diameter of a few hundred nm can be seen at the bottom of the coating (Fig. 3). Some of them are closed, others form interconnected network. Those pores arise during the 
initial stage of the MAO process, while the molecular oxygen is formed at the interface between coating and electrolyte [6]. Furthermore, crater-like pores, with diameter up to $2 \mu \mathrm{m}$ occur, as an effect of the discharges during the process operated above the breakdown voltage of growing oxide layer [6].

To understand how cells interact with biomaterial microscopic observations are performed after in vitro cell culture. Cell line MG-63, which was well adapted to the rough and porous surface of the coating is presented in Fig. 4a. The cells spreading at the material as well as the formation of cytoplasmic protrusions such as filopodia were observed indicating that the provided surface is suitable for their growth and migration [4]. Analysis of the interface between cell and material requires the cross-section approach. To investigate the interior of the sample composed of soft and delicate cell on the hard surface of porous, ceramic coating we performed FIB milling and subsequent SEM imaging. The "sliceand-view" technique by FIB-SEM tomography enables to analyse the cell-material interface not only at one point, but in scanned volume. MG-63 cell was perfectly adjusted to the topography of the outer part of the coating composed of HA crystals, as presented at the crosssection image (Fig. 4b) as well as in the $3 \mathrm{D}$ reconstruction (Fig. 5).

FIB-SEM tomography is widely used to investigate biological samples embedded in resin, and it allows to visualize the internal location of cellular organelle in 3D [14]. However, the application of this technique in cell-biomaterial interactions is just currently being explored [2-5]. With the 3D reconstruction of investigated volume $(9 \times 3.6 \times 4 \mu \mathrm{m})$, it was possible to confirm the excellent spreading of the cell at the ceramic coating (Fig. 5) as well as adjustment to the nanorough topography. Platelet-like shape HA in the outer part of the coating can mimic the native extracellular matrix (ECM) and provide high surface area for enhanced cells adhesion.

\section{Conclusions}

MAO coating was characterized in 3D by FIB-SEM tomography, showing porosity of $24 \%$. Reconstruction of analysed volume enabled detailed investigation of layered structure of the coating and visualization of internal pores. Highly porous, rough, and HA containing surface was found to promote cells adhesion, spreading and filopodia formation. Additionally, by means of FIB-SEM tomography the $3 \mathrm{D}$ visualization of the coherent interface between the cell and ceramic coating was done.

\section{Acknowledgments}

The study was realized within OPTYMED research project (no. 2013/08/M/ST8/00332) financed by National Science Centre of Poland.

\section{References}

[1] K. Duan, R. Wang, J. Mater. Chem. 16, 2309 (2006).

[2] U. Stachewicz, T. Qiao, S.C.F. Rawlinson, F.V. Almeida, W.Q. Li, M. Cattell, A.H. Barber, Acta Biomater. 27, 88 (2015).

[3] A. Fredmann, A. Hoess, A. Cismak, A. Heilmann, Acta Biomater. 7, 2499 (2011).

[4] H.T. Chen, C.J. Chung, T.C. Yang, C.H. Tang, J.L. He, Appl. Surf. Sci. 266, 73 (2013).

[5] T.C. Yang, H.Y. Shu, H.T. Chen, C.J. Chung, J.L. He, Surf. Coat. Technol. 259, 185 (2014).

[6] A.L. Yerokhin, X. Nie, A. Leyland, A. Matthews, S.J. Dowey, Surf. Coat. Technol. 122, 73 (1999).

[7] M. Zientara, A. Kruk, A. Gruszczynski, A. CzyrskaFilemonowicz, Mater. Character. 87, 143 (2014).

[8] T. Moskalewicz, A. Kruk, M. Kot, S. Kayali, A. Czyrska-Filemonowicz, Arch. Civil Mech. Eng. 14, $370(2014)$

[9] J.T.Y. Lee, K.L. Chow, Scanning 34, 12 (2012).

[10] V. Lešer, D. Drobne, Ž. Pipan, M. Milani, F. Tatti, J. Microsc. 233, 309 (2009).

[11] P. Moran, B. Coats, Microsc. Today 20, 28 (2012).

[12] J. Karbowniczek, S. Metwally, G. Cempura, A. Czyrska-Filemonowicz, Key Eng. Mater. 687, 106 (2016).

[13] Y. Zou, J. Malzbender, Ceram. Int. 42, 2861 (2016).

[14] C. Kizilyaprak, J. Daraspe, B.M. Humbel, J. Microsc. 254, 109 (2014). 